\title{
Technology to Support Rural Health and Well-being
}

\author{
Leila Eadie, Alasdair Mort, Philip Wilson \\ Centre for Rural Health, University of Aberdeen \\ Centre for Health Science, Inverness IV2 3JH \\ l.eadie@abdn.ac.uk
}

\begin{abstract}
Recent technology research and implementation initiatives have aimed to ensure equivalent healthcare access and outcomes for people living in remote and rural areas to those obtained by urban residents. This short paper describes three projects undertaken in the Highlands of Scotland at the Centre for Rural Health before moving on to discuss potential future research ideas generated by an interactive workshop.
\end{abstract}

Keywords: rural, health, pre-hospital, emergency care, telemedicine, data transmission.

\section{INTRODUCTION}

Problems associated with both distance and lack of infrastructure can disadvantage people living in remote and rural areas compared to urban dwellers when it comes to care and support for their health and well-being. Equitable care should be attainable in this modern era through communications and health technology, but this is rarely achieved.

\section{CURRENT RESEARCH EXAMPLES}

There is a range of current research in the area of technology use for rural healthcare support ongoing in the Scottish Highlands, and this article will provide a brief summary of several of these projects, followed by a future-looking discussion of currently unmet needs in this area.

The Implementing Transnational Telemedicine Solutions (ITTS) [http://www.transnationaltelemedicine.eu] project is a multinational EUfunded study which aimed to implement telemedicine solutions, at scale, normalising the use of technology into everyday practice [1]. It addressed the challenges of an ageing and geographically-dispersed population, encouraging the use of telemedicine in Europe's Northern Periphery with partners from Norway, Finland, Sweden, Republic of Ireland and Northern Ireland. It did this through transnational knowledge exchange and improving accessibility by situating services in local communities or in patients' homes. ITTS is currently at the reporting stage, ready to share learning about how transnational knowledge exchange facilitated the implementation of telemedicine applications in remote and rural areas.

Ten demonstrator projects on the themes of videoconsultation (VC), mobile self-management and home-based health services were implemented across a wide range of clinical specialities, including:

- Videoconferencing for speech therapy, renal medicine, emergency psychiatry and remote diabetes services;

- Mobile health applications for tracking physical activity and inflammatory bowel disease;

- Home-based services for remote exercise classes for rehabilitation, support in medical and social care emergencies and care for patients with multimorbidity.

The strategy was to learn from successful established telemedicine services in Northern Europe and to introduce these in other areas, to build sustainable solutions. The aim was therefore not to create new technology, but to apply existing approaches: helping people to make use of healthcare equipment and options that were already available. In this way, ITTS supported the development of 25 new services at more than 40 sites in the six participating countries. Results will inform the creation of a guide featuring an interactive checklist for use by policymakers and service planners considering implementing telemedicine systems. A project using a mixture of old and new technology is Satellite Ultrasound for Rural Stroke (SURS), which is testing and developing protocols to stream live transcranial ultrasound imaging from ambulances in the field to offer early diagnosis for people suffering from stroke [2]. The outcome for many stroke patients could potentially be improved if they receive timely treatment; however, it can take a considerable period of time for an ambulance to reach patients then transport them to a centre of definitive care. As such, rapid response to stroke is problematic in remote and rural areas worldwide. 
The focus of SURS is on the early, rapid assessment of patients in the pre-hospital environment (e.g., by paramedics or ambulance clinicians), using remotely-supported point of care ultrasound (RSPOCUS) culminating in an expert diagnostic assessment. If this can be done safely and reliably then pre-hospital treatment options could be possible in future. Installing ultrasound and image transmission/videoconferencing equipment in ambulances could have wide applicability in supporting paramedics to make a diagnosis across various emergency and other situations, such as in trauma, lung and cardiac problems.

The main problem to be overcome is the real time data transmission in areas that are notoriously signal-poor, and researchers are helping to optimise a communications system that uses both cellular and satellite networks to transmit data to a distant location for real-time review. Initial data from field studies where images were sent from sites across the Highlands to a remote clinician have shown that such transmission is possible (using enhanced aerials), and over $90 \%$ of images were rated as being are of diagnostic quality.

Another project aiming to support pre-hospital care is Managing Information in Medical Emergencies (MIME) $^{1}$, which has developed an innovative technology platform to facilitate the recording and handover of patient data by Community First Responders (lay-people with basic first aid training and limited equipment, who assess the patient and their situation, sometimes carrying out simple emergency procedures and/or seeking additional help as appropriate) [3].

This includes prompting for vital signs, immediate management actions and observations about the patient's condition, and uses Natural Language Generation to automatically create a handover report based on the data recorded. Some support for decision making is also planned, using key features in the patient history, clinical measurements and trends over time, safe limits for these parameters beyond which alarms should be activated, and actions that should be instituted on the basis of these parameters.

The aim is to create an efficient, useful interface to support pre-hospital management, which can be personalised for local protocols and generate useful data summaries for the transfer of patients to definitive care. A fully functional prototype has now been tested in the field with pre-hospital patients, and development is ongoing to incorporate more sensors.

\section{DISCUSSION}

These are just three projects using varying levels of novel and established technology to enhance the options for rural and remote healthcare. They rely on relatively simple ideas, which when discussed with communities tend to provoke the question: "aren't we doing that already?"

This demonstrates that unmet needs do not have to be complex, requiring new high-tech equipment to be designed; sometimes, providing basic support to first-line health responders can be enough to help streamline services and improve the situation for all involved.

So, where else can technology be applied to help those living in or providing a service to rural areas?

A workshop forming part of the 2014 BCS Health Informatics Scotland annual conference ${ }^{2}$ initiated a discussion about how we can support people with chronic conditions to continue living in remote and rural areas, yet still receive care equal to those living in urban areas; and whether current technologies are adequate to facilitate the delivery of time-critical care in pre-hospital remote and rural emergencies. Two main foci for future development were suggested.

\subsection{General Practitioner Support}

If we can provide video communications facilities to ambulances, then why not also to GPs, to allow them better access to support from secondary care experts? More videoconferencing services are moving into rural communities - for example, regular consultations with diabetes specialists - so it seems feasible that GP surgeries could each have their own system to connect them with a centre of definitive care to help with queries that are out of the scope of primary care and provide another form of prehospital patient triage.

\subsection{Emergency Support}

A simple suggestion to improve pre-hospital emergency care is to provide paramedics with access to patients' summary care records. This could readily be done via a communications link to a hospital, similar to that used in SURS, which can be the gatekeeper for the information. Data privacy issues would need to be addressed, but this data access could prove very useful to emergency care personnel, which was the intent behind the record's original conception.

\footnotetext{
${ }^{1}$ http://www.dotrural.ac.uk/mime/
} 


\section{CONCLUSIONS}

In this age of ubiquitous communication and technology, we should be able to support people living in remote and rural regions so that the care they receive is on a par with that received in the city, close to health services. It is clear that simple additions to, or new uses for, standard equipment can help improve the situation, and yet there is still more work to be done. They key is to provide timely access to the right information at the location where it is needed, and this is something that rapidly progressing technology can facilitate.

\section{ACKNOWLEDGEMENTS}

We would like to thank the participants in the workshop on which this article is based, for their engagement and ideas in the lively discussions. We would also like to acknowledge the support of the University of Aberdeen's dot.rural Digital Economy Hub (RCUK Digital Economy programme reference EP/G066051/1), Highlands and Islands Enterprise, and the Scottish Ambulance Service in particular.

\section{REFERENCES}

[1] Casey, M., Hayes, P.S., Heaney, D., Dowie, L., Olaighin, G., Matero, M., Hun, S., Knarvik, U., Alrutz, K., Eadie, L., Glynn, L.G. (2013) Implementing transnational telemedicine solutions: A connected health project in rural and remote areas of six Northern Periphery countries. European Journal of General Practice, 19, 52-58.

[2] Mort, A.J., Eadie, L., Regan, L., Macaden, A., Heaney, D., Bouamrane, M.M., Rushworth, G.F. \& Wilson, P. (in press) Combining transcranial ultrasound with intelligent communications methods to enhance the remote assessment and management of stroke patients - Framework for a technology demonstrator. Health Informatics Journal.

[3] Schneider AH, Mort AJ, Kindness P, Mellish C, Reiter E, Wilson P. (in press) Using technology to enhance rural resilience in pre-hospital emergencies. Scottish Geographical Journal. 